\title{
Analysis on the Network Structure of Public Opinion transmission about social hot issues based on the microblogging platform
}

\author{
Yangbing Yanga, ${ }^{\mathrm{k}}$, Jianping Chai ${ }^{\mathrm{b}}$, Bo $\mathrm{Li}^{\mathrm{c}}, \mathrm{Xi}$ Feng ${ }^{\mathrm{d}}$, \\ Zhuang Wange \\ School of Information \&Engineering, Communication University of China, Beijing 10024, China; \\ a862165191@qq.com, bjp_chai@sina.com, clbmovie@sina.com, dfengxi@cuc.edu.cn, \\ e540062754@qq.com
}

Keywords: network structure, big data, hot issues, Ucinet

\begin{abstract}
At present, there are a large number of Internet users in the microblogging to express their views, they are associated with each other by forwarding. This paper presents a method about an analysis on the network structure of public opinion transmission about social hot issue based on the microblogging platform. To "CCTV Spring Festival Evening" as an example, we use social network analysis methods, use web crawler to get data, use "Ucinet" to calculate the central, intermediary and other data of the network structure, and draw a picture of the network structure of the transmission. So we can research the characteristics of network structure and key node recognition of public opinion transmission in hot issues.
\end{abstract}

\section{Introduction}

With the social media into the tens of thousands of households, everyone has a platform to express their views, the citizens has paid more attention to the speaking right. At the same time, in the era of large data, social hot events have some characteristics, for example, they are often difficult to predict; they have large impact and serious consequences; they are often complex and highly sensitive; and so on. Network public opinion is a reflection of real problems on Internet. Improper handling will cause a lot of adverse reactions, affecting social stability. Therefore, it is more important to analyze the network structure of public opinion transmission about social hot issues.

In the academic community, the analysis on the network structure of public opinion transmission attracts widespread concern of communication, sociology, management, mathematics and communication and other multidisciplinary scholars. Theoretical research in this area of social networks is generally considered to begin with Mllgram's famous "six-degree division"[1]. From the aspects of crisis attributes, public coping experience, gender, social orientation and so on. Helsloot and Ruitenberg analyzed the psychological and behavioral impact of crisis information on different groups. Berelson believes that communication is the transmission of information and is not necessarily accepted or understood [2]. In 1973, Granovetter also put forward the classic "weak connection" theory, the focus of its research is putting personal behavior in interpersonal interaction network observation. [3]. in 1998, in Cornell University, Watts and Strogat expressed the small world character of social networks and established a small world network model [4]. In 2003, Elgazzar defined the evolution rules [5]. In recent years, the academic community has further integrated the analysis of the network with the transmission of crisis information. Naim Kapucu proposed a network analysis to assess the relationship between responding organizations and coordinate emergency action. Harikesh used natural experiments in 2010 to present new guidelines for the impact of network behavior and to fully emphasize the role of opinion leaders [6]. The representative research of domestic scholars includes the following aspects: According to the method of node deletion, Pengxiang Li puts forward the evaluation method based on the importance of weighted network and weighted network nodes [7]. Jin Xu proposed the "nuclear degree theory", which is based on the "node importance is equal to the node and other nodes connected relationship" [8]. Lang Liu proposed the importance of the node 
priority calculation method. Dajun Zeng proposed a propagation model based on heterogeneous network structure [9]. Binxing Fang proposed an event-based social network evolution analysis framework and conducted a study on public opinion monitoring [10]. In 2009, viewing the evolution of public opinion as the object, Guodong Zhu analyzed network structure characteristics, such as full connectivity, small world model and the influence of scale-free characteristics on the propagation speed of topic and the stability of group view [11]. In 2013, to "0723 - motor vehicle accident" as an example, Wei Kang used social network analysis of the network transmission structure and key point of identification, to explore the public opinion of the network structure, the influence of node location and the relationship between each nodes to the information propagation path, propagation speed and propagation range [12].

Today, public opinion analysis pays more and more attention to the combination of theory and practice. To "CCTV Spring Festival Evening" as an example, we use social network analysis methods, use web crawler to get data, use "Ucinet" to calculate the central, intermediary and other data of the network structure, and draw a picture of the network structure of the transmission. So we can research the characteristics of network structure, key node recognition of public opinion transmission in hot issues, and find the influence of the location of the key node on the transmission of public opinion in the network.

\section{Intelligent optimization algorithm model for film schedule}

When the network structure is drawn by the social network analysis theory, we should calculate important parameters that represent weak connections in the propagation structure, such as central, intermediary. So that the influence of key nodes can be found and the analysis result can be more accurate and scientific.

\subsection{Network density measurement.}

Network density measurement, also known as microblogging public opinion network density, refers to the network structure used to measure the shortest path between the two different nodes. Network density is the degree of information communication between the members of the network, Depending on the network structure parameters. The measurement of density needs to check the number of existing social network connections and the maximum number of existing social network connections. And the value is between $0-1$. The closer the value is to 0 , the more loose the relationship between each other. The closer the value is to 1 , the closer the relationship is. The density calculation reflects the positive degree of participation of members in the exchange. For an undirected graph containing $\mathrm{n}$ points, the maximum number of possible connections is $\frac{\mathrm{n}(n-1)}{2}$, so we get the formula for the density of undirected graphs as follows:

$$
\mathrm{D}=\frac{2 l}{n(n-1)}
$$

Where 1 is the actual number of connections for the social network graph (ie, the sum of all the boundary values in the value graph), and $\mathrm{n}$ is the number of nodes in the social network graph.

\subsection{Central.}

In this part, the commonly used parameters are dot center and normalized dot center. Dot center, also known as the degree of central, describes the ability to a node with other nodes directly interact and contact. For nodes with strong direct interactivity, their dot center degree is higher, which indicates that the node is closer to the center of the social network communication structure. The degree of dot center is divided the degree of entering point and the degree of leaving point. The degree of entering point refers to the sum of the boundary values directly pointing to the node. The degree of leaving point is the sum of the boundary values directly from the node to other nodes. In this point, the degree of leaving point mainly measures the ability of the node to influence others, and the degree of entering point is mainly measured by the ability of accepting others impaction. We get the formula for the degree of dot center as follows: 


$$
C_{D} n_{i}=\sum_{j} X_{i j}
$$

$X_{i j}$ is 0 or 1 , it means whether node $\mathrm{j}$ is communicating with node $\mathrm{i}$, and $\mathrm{n}$ is the number of nodes in the entire network.

The normalized dot center is the result of the standardization of dot center, and it can also be divided into the standardization degree of entering point and the standardization degree of leaving point, which reflects the concentrated trend of the node. So we get the formula for the degree of normalized dot center as follows:

$$
C_{D} n i=\frac{\sum_{i=1}^{n}\left(C_{D \max }-C_{D i}\right)}{\max \left[\sum_{i=1}^{n}\left(C_{D \max }-C_{D i}\right)\right]}
$$

$C_{D i}$ is the dot center of the $\mathrm{i}$-th node, $C_{D \max }$ is the maximum value of $C_{D i}$ for all nodes, and $\mathrm{n}$ is the number of nodes in the entire network.

\subsection{Intermediary.}

when one node is viewed as an intermediate node, the intermediary center is the ability of to propagate and the number of times that is occupied in the shortest path between the other two nodes ,that is, the measurement node is the capacity of the existing bridge. This represents the "bridge" of the shortest path junction between two or more nodes, and the higher the number of nodes is, the more the node must touch. The corresponding formula for the intermediary center is as follows:

$$
C_{B}=\frac{\sum_{j<k} g_{j k}(n i)}{g_{j k}}
$$

$g_{j k}$ is the sum of the shortest number of routes between points $\mathrm{j}$ and $\mathrm{k}$, and $g_{j k}(n i)$ represents the shortest number of routes between the two actions that contain the actor $n i$.

The normalized intermediary center is the difference between the node with the highest degree of intermediary in the network structure and the intermediary degree of the other nodes. The higher the gap between the node and other nodes, the higher normalized intermediary center of the network is. The corresponding formula for the normalized intermediary center is as follows:

$$
C_{B}=\frac{2 \sum_{i}^{N}\left(C_{B \max }-C_{B i}\right)}{n-1^{2}(n-2)}
$$

$C_{B i}$ is the normalized intermediary center of the i- th node, and $C_{B m a x}$ is the maximum of $C_{B i}$.

\section{Case Analysis}

Select the "2016 CCTV Spring Festival Evening" as an example. Grab the microblogging of "CCTV news", "days of entertainment media" and other large V published on the 2016 CCTV Spring Festival. There are about 20 microblogging, and each microblogging crawl about 1500 data. Using network crawler technology collects the forwarding text on microblogging. Using $\mathrm{R}$ language programming procedures for data preprocessing, we can get related microblogging forwarding relationship node about hot events. Then we use Excel software to complete Perspective analysis of the data. Finally, use Ucinet software. The software can calculate some parameters such as central, and it can analysis the influence between the microblogs, establish the communication relation model and draw the picture of network structure.

This article provides Microblogging forwarding rules: if the user does not publish any comments when the view, then this will be forwarded from the original microblogging; if the user publish some text content when forwarding, and the text includes the style of "@**", then forward from the @ after the person, otherwise it is forwarded from the original microblogging. However, for "H; @ F @ B @ A", then look at the front of the forwarding relationship directly. That $\mathrm{H}$ forwarded the microblogging F. 
Using the methods of calculating of central and intermediary mentioned above, the key nodes obtained are calculated one by one. The following figure shows the specific calculation of more influential microblogging accounts on this hot event.

From the results, we can find the influence of "CCTV news"is higher than the influence of "Day entertainment media", the influence of "Zhang Jie DreamWorks". Note that in the incident on the Spring Festival Evening, CCTV news with its unique advantages is in an absolute dominant position about public opinion occupies.

\begin{tabular}{|c|c|c|c|c|c|}
\hline 些 - & $\times$ & 哋 & $\times$ & 祡一 & $\square$ \\
\hline Key & Value & Key & Value & Key & Value \\
\hline ID & CCTV news & ID & ainment media & ID & DreamWorks \\
\hline Mode & Columns & Mode & Columns & "Mode & Columns \\
\hline Degree & 42 & Degree & 22 & Degree & 13 \\
\hline Betweenness & 5002.000 & Betweenness & 2772.000 & Betweenness & 1638.000 \\
\hline Closeness & 482.000 & Closeness & 522.000 & Closeness & 540.000 \\
\hline HarmonicClosı & 76.833 & HarmonicClosı & 63.500 & HarmonicClosı & 57.500 \\
\hline Eigenvector & 0.180 & Eigenvector & 0.011 & Eigenvector & 0.008 \\
\hline 2-Local Eigen & 49.000 & 2-Local Eigen & 29.000 & 2-Local Eigen & 20.000 \\
\hline
\end{tabular}

Fig 1. Data calculation of "CCTV news", "Day entertainment media", "Zhang Jie DreamWorks"

In the past, the analysis of microblogging propagation structure only choose one information dissemination network, the results have a one-sided problem. However, this article collected a number of representative of the popular microblogging about a single event .And single microblogging information dissemination network can be integrated for the whole topic of a large network, so that the results more scientific and objective. For the CCTV Spring Festival Evening to capture all the microblogging, its forwarding relationship network structure can be shown in Figure2.

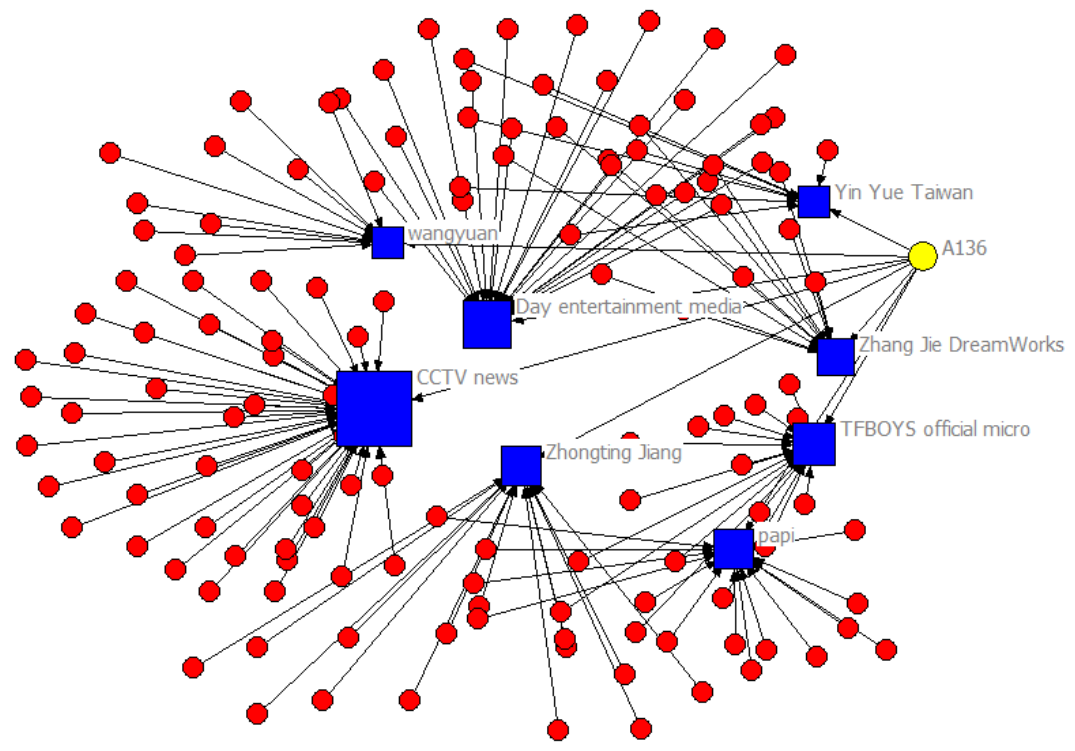

Fig 2. The network structure picture of CCTV Spring Festival

From the above figure, we can observe that: For a few large blue points ,it means a lot of people forwarded this type of account microblogging, including CCTV news, star fans fan club and so on. For yellow spots, this means that a group of people who are particularly interested in forwarding microblogging. This group is characterized by a small number of fans, they want to improve their influence. What's more they often concern about the hot events, often published their views, but they are also vulnerable to the views of others, such as the water army.

\section{Conclusion}

This paper puts forward using social network analysis method, combining the traditional graph theory and computer science, we can achieve the network public opinion communication structure 
analysis interdisciplinary. We can find the influence of the location of Key Nodes in the Network on the Propagation Path and Propagation Velocity of public opinion information. When faced with emergencies, based on the role of different nodes, public opinion managers can get related theoretical and practical guidance, so as to control the evolution of public opinions. And provide reference for policymakers to control public opinion. In the future, we can further study the identification of opinion leaders and key nodes, and how to introduce the network public opinion communication structure relationship into the network public opinion evaluation system.

\section{References}

[1]. Wenhao Huang, Haikun Hong, Guojie Song, Kunqing Xie .Deep process neural network for temporal deep learning [C].Neural Networks (IJCNN), 2014: 465- 472.

[2]. Angiulli, G.,Cacciola, M. ,Versaci, M. Microwave Devices and Antennas Modelling by Support Vector Regression Machines [C].Magnetics, 2007: 1589-1592..

[3]. Renqiang Min, Stanley, D.A., Zineng Yuan,Bonner, A., Zhaolei Zhang . A Deep Non-linear Feature Mapping for Large-Margin kNN Classification [C].Data Mining, 2009: 357- 366.

[4]. T.K.Das, D.P.Acharjya, M.R.Patra, Opinion mining about a product by analyzing public tweets in Twitter [C] .Computer Communication and Informatics (ICCCI), 2014,1-4

[5]. Jianhua Ruan and Weixiong Zhang. An efficient Spectral Algorithm for Network Community Discovery and Its Applications to Biological and Social Networks [C]. Proceedings of the 2007 Seventh IEEE International Conference on Data Mining, 2007, 643 648.

[6]. Qin, Sheng. A web-based distributed group decision support system for railway construction organization [J]. Informatics in Control, Automation and Robotics (CAR), 2010 2nd International Asia Conference on, 2010, (3): 362-365.

[7]. Weidong li, Pengxiang Li. Complex network topology model of microblogging public opinion and its evolution mechanism [J]. News and communication research. 2013(11).

[8]. Yi Liu. Network public opinion research [M]. Tianjin. Tianjin People's Publishing House

[9]. Lang Liu, Yi Liu. On the concept, characteristics, expression and dissemination of network public opinion [J]. Theories, 2007, 2007 (1): 11-12

[10]. Deshun Li. A Study on the Formation of Public Opinion in the Age of Media Convergence The Game of Public Power in Public Domain [D]. Fudan University. 2013.

[11]. Shaohua Qing, Peng Wu. Research on the Simulation of Internet Users' Group Behavior in the Process of Public Opinion Evolution [J] . Modern Library and Information Technology, 2014, 30 (6): $71-78$

[12]. Wei Kang. dentification of Key Points of Public Opinion in Emergency Network Based on SNA - Taking 7.23 Motor Vehicle Accident as an Example [J]. Journal of Public Administration. 2012 (7). 